\title{
Abbreviations and Acronyms
}

\begin{tabular}{|c|c|}
\hline AAT & Altech Telecommunications Ltd \\
\hline $\mathrm{ADH}$ & Asynchronous Digital Hierarchy \\
\hline ADSL & Asynchronous Digital Subscriber Line \\
\hline AFRALTI & African Advanced Level Telecommunication Institute \\
\hline ANOVA & Analysis of Variance \\
\hline ATM & Asynchronous Transfer Mode \\
\hline ATU & African Telecommunication Union \\
\hline AXE & $\begin{array}{l}\text { Ericsson family of telecommunication networking } \\
\text { equipment }\end{array}$ \\
\hline CCITT & $\begin{array}{l}\text { Commité Consultatif International Télégraphique et } \\
\text { Téléphonique (an ITU committee) }\end{array}$ \\
\hline CLI & Caller Line Identification \\
\hline CNET & $\begin{array}{l}\text { Centre National d'Etudes des Télécommunications: } \\
\text { National Center for Telecommunications Studies } \\
\text { (France) }\end{array}$ \\
\hline CSIR/STEPRI & $\begin{array}{l}\text { Council for Scientific and Industrial Research/Science } \\
\text { and Technology Policy Research Institute }\end{array}$ \\
\hline CSF & Critical Success Factor \\
\hline CTO & Commonwealth Telecommunication Organization \\
\hline DECT & Digital European Cordless Telecommunications \\
\hline DTM & Digital Terrain Model \\
\hline ESRF & Economic and Social Research Foundation \\
\hline ETSI & European Telecommunication Standards Institute \\
\hline FCC & Federal Communications Commission (US) \\
\hline FDI & Foreign Direct Investment \\
\hline GIS & Geographic Information System \\
\hline GMPCS & Global Mobile Personal Communication Service \\
\hline GPRS & Global Packet Radio Service \\
\hline GSM & Global System for Mobile Service \\
\hline HIPC & Highly Indebted Poor Countries Initiative \\
\hline HRD & Human Resource Development \\
\hline IDD & International Direct Dialling \\
\hline ICT & Information and Communication Technology \\
\hline ISDN & Integrated Services Digital Network \\
\hline IT/IS & Information Technology/Information Systems \\
\hline ITU & International Telecommunication Union \\
\hline JIT & Just in Time \\
\hline
\end{tabular}


JV

MIS

NCA

NGO

NIS

NTT

OECD

Oftel

PABX

PARUC

PATU

PTO

R\&D

SATRA

SC

SDH

SETA

SMME

SNO

TCC

TCP/IP

TDMA

TNC

TNS

TSh

TT

UCC

UTL

UMTS

UNDP

USTTI

Westel

WAN/LAN

WLL

Z-Comms
Joint Venture

Management Information Systems

National Communications Authority

Non Governmental Organization

National Innovation System

Nippon Telegraph and Telephone Corporation

Organisation for Economic Cooperation and

Development

Office of Telecommunication (UK)

Private Automatic Branch Exchange

Pennsylvania Regulatory Utilities Commission

Pan-African Telecommunication Union

Public Telecommunication Operator

Research and Development

South African Telecommunications Regulatory

Authority

Strategic Competence

Synchronous Digital Hierarchy

Sector Education and Training Authority

Small, Medium and Micro Enterprise

Second National Operator

Tanzania Communications Commission

Transmission Control Protocol/Internet Protocol

Time Division Multiple Access

Trans-National Corporation

Telecommunication Network-based Services

Tanzania Shilling

Technology Transfer

Uganda Communications Commission

Uganda Telecommunication Ltd

Universal Mobile Telephone Service

United Nations Development Programme

United States Telecommunication Training Institute

ACG Systems (Ghana) Ltd

Wide Area Network/Local Area Network

Wireless Local Loop

Zakheni Communications Strategies 